\title{
The Chaotic Properties of Increasing Gap Shifts
}

\author{
Nor Syahmina Kamarudin (D), Malouh Baloush (D), and Syahida Che Dzul-Kifli $(\mathbb{D}$ \\ School of Mathematical Sciences, Faculty of Science and Technology, Universiti Kebangsaan Malaysia, 43600 UKM Bangi, \\ Selangor, Malaysia \\ Correspondence should be addressed to Syahida Che Dzul-Kifli; syahida@ukm.edu.my
}

Received 30 July 2018; Revised 7 November 2018; Accepted 28 January 2019; Published 27 February 2019

Academic Editor: Frédéric Mynard

Copyright (C) 2019 Nor Syahmina Kamarudin et al. This is an open access article distributed under the Creative Commons Attribution License, which permits unrestricted use, distribution, and reproduction in any medium, provided the original work is properly cited.

\begin{abstract}
It is well known that locally everywhere onto, totally transitive, and topologically mixing are equivalent on shift of finite type. It turns out that this relation does not hold true on shift of infinite type. We introduce the increasing gap shift and determine its chaotic properties. The increasing gap shift and the sigma star shift serve as counterexamples to show the relation between the three chaos notions on shift of infinite type.
\end{abstract}

\section{Introduction}

The main ingredients for Devaney chaos is topologically transitive, dense periodic points, and sensitive dependence on initial conditions as originally defined by Devaney [1]. Later, it turns out that the third condition is redundant and therefore being excluded from the definition afterwards [2]. Even though other definitions of chaos with different senses were introduced, Devaney chaos has received more attention among the others. See Guirao et al. [3] for different versions of chaos definitions. On top of that there are more chaos notions that have been brought up to expose more characterizations of dynamical systems that exhibit randomness behavior. The incomplete list of the chaos notions is totally transitive, topologically mixing (or mixing), blending, locally everywhere onto (shortly l.e.o and also known as topologically exact), specification property, strong dense periodicity property and expansive. Following the introduction of many chaos notions, researchers started to find connections between those notions on variety topological spaces. Thomson [4] formed a hierarchy of three chaos notions on the interval, circle, torus and sphere. Li and Ye [5] had listed some recent development of chaos theory in topological dynamics by focusing on some versions of chaos with their relationships. See Fan et al. [6], Crannell [7], Alseda et al. [8], Denker et al. [9], Syahida and Good [10], and Malouh and Syahida [11] for relations on graph maps, shift spaces, closed interval, etc.
It is well known that having the property of transitivity is sufficient enough for a system on the interval [12] and, on the infinite shift space, it is obvious that transitivity implies dense periodic points and therefore implies SDIC [2]. This is not true if we replace the property of transitivity with the other ingredient of Devaney chaos, dense periodicity property. It turns out, however, that strengthening this dense periodicity property yields different results. On shift of finite type, the implication is true [13] but not the case on the unit interval [10]. The study of chaotic behavior on shift of finite type has been done extensively in various approaches. However, analogues to results on shift of finite type are not much explored on shift of infinite type even though the main gaps between these two spaces are finiteness of its number of forbidden blocks. It is well known that totally transitive, topologically mixing, l.e.o., and specification property are equivalent on shift of finite type (see Crannell [7] and Fan et al. [6]). These equivalences are not necessarily true on general. At least Thomson [4] has provided an example on the interval with topologically mixing and totally transitive properties but without locally everywhere onto, while Ruette [14] has given an example that there is an interval map which is topologically mixing but it is not locally everywhere onto. The sigma star (see [7]) is another counterexample on shift of infinite type to demonstrate this phenomenon. In this work, we will consider a certain example of shift of infinite type and discuss its dynamical properties in order to see the differences 
between relations among these chaos notions on these two types of shift spaces.

In the other perspective, Baker and Ghenciu [15] study the dynamical properties of certain $S$-gap shift and introduce two shift spaces: boundedly supermultiplicative (BSM) shifts and balanced shifts. Dawoud and Somaye [16] also look at topological dynamics of $S$-gap shift in terms of almost finite type, eventually constant, sofic etc.

\section{Preliminary}

To define shift space and its subspaces (shift of finite type and shift of infinite type), we let $A_{n}=\{0,1,2, \ldots, n-1\}$ be the alphabet set of $n$ symbols. Consider the full $n$-shift; $\sum_{n}=\left\{\mathbf{x}=\left\{x_{k}\right\}_{k \in \mathbb{N}_{0}} \mid x_{k} \in A_{n}\right\}$ as the collection of all sequences over symbols in $A_{n}$. The shift map $\sigma$ on $\sum_{n}$ is defined as $\sigma\left(x_{0} x_{1} x_{2} x_{3} \ldots\right)=\left(x_{1} x_{2} x_{3} \ldots\right)$. We call $=w_{0} w_{1} \ldots w_{l-1}$, where $w_{i} \in A_{n}$ for every $i=0,1,2, \ldots, l-1$ as a block of length $l$. For a block $w$, we denote $C_{w}$ as a collection of any sequence in $\sum_{n}$ which started with the block $w$, i.e., $C_{w}=\left\{\mathbf{x}=x_{0} x_{1} x_{2} x_{3} \ldots \in \sum_{n} \mid x_{0} x_{1} x_{2} \ldots x_{l-1}=\right.$ $\left.w_{0} w_{1} w_{2} \ldots w_{l-1}\right\}$. The set $C_{w}$ is called a cylinder set. The metric on $\sum_{n}$ is $d(\mathbf{s}, \mathbf{t})=1 / 2^{j}$, where $j$ is the first entry of $\mathbf{s}$ and $\mathbf{t}$ in $\sum_{n}$ such that $s_{j} \neq t_{j}$. The family of cylinder sets $C_{w}$ where $w$ is any allowed block form a basis for a topology on full $n$-shift, $\sum_{n}$ induced by a metric $d$.

A closed subspace $X$ of $\sum_{n}$ is called a shift space if it is invariant under the map $\sigma$. We may see that, for every shift space, there is a set $F$ of forbidden blocks where $X$ is a collection of any sequences in $\sum_{n}$ which do not contain any block in $F$. We may then denote $X$ as $X_{F}$. If the set $F$ is finite, then $X_{F}$ is shift of finite type and vice versa. Therefore, shift of finite type and its complement, shift of infinite type, are just distinguished based on the finiteness of the number of forbidden blocks but we found that they have different chaotic properties. Crannell [7] introduced the sigma star as the following set: $\sum_{*}=\left\{\left\{x_{0} x_{1} x_{2} x_{3} \ldots\right\} \in \sum_{3} \mid x_{i}=0, x_{j}=\right.$ $2 \Longrightarrow|i-j| \neq 2^{p}$, for all $\left.p=0,1,2, \ldots\right\}$, and it is a shift of infinite type. It is shown that $\left(\sum_{*}, \sigma\right)$ is totally transitive and strongly blending but not topologically mixing.

A dynamical system $\left(X_{F}, \sigma\right)$ is said to be topologically transitive if, for any pair of open sets $U$ and $V$, there exists an integer $n \geq 1$, such that $\sigma^{n}(U) \cap V \neq \emptyset$ and it is totally transitive if $\sigma^{n}$ is topologically transitive for all positive integers $n \geq 1$. Mixing is when, for every pair of nonempty open subsets $U, V \subset X_{F}$ there exists an integer $N \in \mathbb{N}$ such that $\sigma^{n}(U) \bigcap V \neq \emptyset$, for all $n>N$ and locally everywhere onto is when, for every open subset $U \subset X_{F}$, there exists a positive integer $n$ such that $\sigma^{n}(U)=X_{F} . \sigma$ is said to be strongly blending if for every pair of nonempty open subsets $U, V \subset$ $X_{F}$ there exists an integer $n>0$ such that $\sigma^{n}(U) \cap \sigma^{n}(V)$ contains a nonempty open subset. A strong dense periodicity property is when, for all $n \in \mathbb{N}$, the set of periodic points of prime period $n$ is dense in the whole system.

It is stated earlier that totally transitive, topologically mixing, l.e.o., and specification property are equivalent on shift of finite type. However, the sigma star is a counterexample on shift of infinite type to cross out mixing from the list of equivalence chaos notions. In the sequel section, the increasing gap shift is introduced and its chaotic dynamical properties are explored to show that totally transitive and l.e.o. is also not equivalence on infinite type shifts.

\section{The Increasing Gap Shift}

The increasing gap shift is an example from a larger class of subshift known in literature as $S$-gap shifts $\Sigma(S)$. To define $S$-gap shift, fix an increasing subset $S$ in $\mathbb{N}_{0}=\mathbb{N} \cup\{0\}$. If $S$ is finite, define $\Sigma(S)$ to be the set of all binary sequences for which 1's occur infinitely often and the number of 0 's between successive occurrences of 1 is an integer in $S$. When $S$ is infinite, we need to allow points that begin or end with an infinite string of 0 's. The increasing gap shift $\Sigma(I)$ is an $S$-gap shift with $S=I=\left\{a_{n} \mid a_{0}=1, a_{n}=a_{n-1}+n\right.$ for every $n \in$ $\left.\mathbb{N}_{0}\right\} \subset \mathbb{N}_{0}$. Since it has infinitely many forbidden blocks, it is shift of infinite type. Chaos properties of $\Sigma(I)$ will be explored. Hence we have the following result.

\section{Theorem 1. $(\Sigma(I), \sigma)$ is topologically mixing.}

Proof. Let $C_{w}$ and $C_{v}$ be basic open subsets of $\Sigma(I)$ for allowable blocks $w$ and $v$ with length $l$ and $k$, respectively. We consider cases for $w$ and $v$. For the first case, let $w=$ $w_{0} w_{1} \ldots w_{l-p_{1}-2} 1 \underbrace{00 \ldots 00}_{p_{1}}$ and $v=\underbrace{00 \ldots 00}_{p_{2}} 1 v_{p_{2}+1} \ldots v_{k-1}$, where $p_{1}$ and $p_{2}$ are both in $I$. Choose $N=l+1$. Let $n>N$ such that $n=N+r=l+1+r$ for some $r \in \mathbb{N}$. For any $\mathbf{y} \in C_{v}$, we may take $\mathbf{x}=\underbrace{w 1 \ldots 11}_{r+1} \mathbf{y} \in C_{w}$ such that $\sigma^{l+r+1}(\mathbf{x})=\sigma^{n}(\mathbf{x})=\mathbf{y} \in C_{v}$. Hence, for every $n>l+1$, $\sigma^{n}\left(C_{w}\right) \cap C_{v} \neq \emptyset$. Therefore, $(\Sigma(I), \sigma)$ is mixing for this case. There are 5 other cases to be considered. In each case, we choose different value of $N$ such that, for every $n=N+r>N$ and for some chosen $\mathbf{y} \in C_{v}$, there exists $\mathbf{x} \in C_{w}$ such that $\sigma^{n}(\mathbf{x})=\mathbf{y} \in C_{v}$. The points $\mathbf{x}$ and $\mathbf{y}$ are chosen differently in each case. All cases for $w$ and $v$, chosen value of $N$, points $\mathbf{x}$ and $\mathbf{y}$ are given in Table 1 . Note that $p_{1}$ and $p_{2}$ are any elements in $I$ whereas $c_{1}$ and $c_{2}$ are not in $I$. For any $c_{1} \notin I$, let $g_{1} \in \mathbb{N}$ such that $c_{1}+g_{1} \in I$. The same goes to $c_{2}$ and $g_{2}$.

Note that since $0 \notin I$, the case for $w$ ending with 1 is one of the cases $w=w_{0} w_{1} \cdots w_{l-c_{1}-2} \underbrace{100 \cdots 00}_{c_{1}}$ for any $c_{1} \notin I$. The same goes for the case when $v$ started with 1 ; it is one of the case for $v=\underbrace{00 \cdots 001}_{c_{2}} 1 v_{c_{2}+1} \cdots v_{k-1}$ for any $c_{2} \notin I$. Therefore, $(\Sigma(I), \sigma)$ is topologically mixing.

The next result verifies that $(\Sigma(I), \sigma)$ is also Devaney chaotic.

Theorem 2. Let $P_{n} \subset \Sigma(I)$ be a collection of periodic points of increasing gap shift with prime period at least $n$. Then $P_{n}$ is dense in $\Sigma(I)$ for any $n \in \mathbb{N}$.

Proof. Let $C_{w}$ be a basic open subset of $\Sigma(I)$ for an allowed block $w=w_{0} w_{1} \ldots w_{l-1}$ in $\Sigma(I)$ and $n \in \mathbb{N}$. We will consider the possibilities for the block $w$, where the first case is $w=$ $\underbrace{00 \ldots 00}_{p_{1}} 1 w_{p_{1}+1} \ldots w_{l-p_{2}-2} 1 \underbrace{00 \ldots 00}_{p_{2}}$ for two elements $p_{1}$ and $p_{2}$ in $I$. For this case, take an element $\mathbf{x}$ in $C_{w}$ such that 
TABLE 1: Cases for blocks $w$ and $v$.

\begin{tabular}{|c|c|c|c|c|}
\hline Block $w$ of length $l$ & Block $v$ of length $k$ & $N$ & $\mathbf{y} \in C_{\mathbf{v}}$ & $\mathbf{x} \in C_{w}$ \\
\hline $\begin{array}{l}w_{0} w_{1} \cdots w_{l-p_{1}-2} \\
\underbrace{00 \cdots 00}_{p_{1}}\end{array}$ & $\underbrace{00 \cdots 001}_{p_{2}} v_{p_{2}+1} \cdots v_{k-1}$ & $l+1$ & Any point & $w \underbrace{11 \cdots 11}_{r+1} \mathbf{y}$ \\
\hline $\begin{array}{l}w_{0} w_{1} \cdots w_{l-p_{1}-2} \\
\underbrace{00 \cdots 00}_{p_{1}}\end{array}$ & $\underbrace{00 \cdots 001}_{c_{2}} v_{c_{2}+1} \cdots v_{k-1}$ & $l+g_{2}+1$ & Any point & $w \underbrace{11 \cdots 1100 \cdots 00 y}_{r+1}$ \\
\hline $\begin{array}{l}w_{0} w_{1} \cdots w_{l-p_{1}-2} \\
\underbrace{00 \cdots 00}_{p_{1}}\end{array}$ & $\underbrace{00 \cdots 00}_{k}$ & $l$ & $\overline{000}$ & $w \underbrace{11 \ldots 11}_{r} \mathbf{y}$ \\
\hline $\begin{array}{l}w_{0} w_{1} \cdots w_{l-c_{1}-2} \\
\underbrace{00 \cdots 00}_{c_{1}}\end{array}$ & $\underbrace{00 \cdots 00}_{p_{2}} 1 v_{p_{2}+1} \cdots v_{k-1}$ & $l+g_{1}+1$ & Any point & $w \underbrace{00 \cdots 00}_{g_{1}} \underbrace{11 \cdots 11}_{r+1} \mathbf{y}$ \\
\hline $\begin{array}{l}w_{0} w_{1} \cdots w_{l-c_{1}-2} \\
\underbrace{00 \cdots 00}_{c_{1}}\end{array}$ & $\underbrace{00 \cdots 00}_{c_{2}} 1 v_{c_{2}+1} \cdots v_{k-1}$ & $l+g_{1}+g_{2}+1$ & Any point & $\begin{array}{c}w \underbrace{00 \cdots 0011 \cdots 11}_{g_{1}} \underbrace{}_{r+1} \\
\underbrace{00 \cdots 00 \mathrm{y}}_{g_{2}}\end{array}$ \\
\hline $\begin{array}{l}w_{0} w_{1} \cdots w_{l-c_{1}-2} \\
1 \underbrace{00 \cdots 00}_{c_{1}}\end{array}$ & $\underbrace{00 \cdots 00}_{k}$ & $l$ & $\overline{000}$ & $w \underbrace{11 \ldots 11}_{r} \mathbf{y}$ \\
\hline
\end{tabular}

$\mathbf{x}=\overline{\underbrace{00 \ldots 001}_{p_{1}} w_{p_{1}+1} \ldots w_{l-p_{2}-2} 1 \underbrace{00 \ldots 0011 \cdots 11}_{p_{2}}}=\bar{w}=\overline{\underbrace{11 \cdots 11}_{n}}$ is a periodic point of prime period greater than $n$. Therefore, $C_{w} \cap P_{n} \neq \emptyset$. The other cases and its chosen periodic points with prime period at least $n$ are presented in Table 2 . Note that $p_{1}$ and $p_{2}$ are any elements in $I$ whereas $c_{1}$ and $c_{2}$ are not in $I$. For any $c_{1} \notin I$, let $g_{1} \in \mathbb{N}$ such that $c_{1}+g_{1} \in I$. The same goes to $c_{2}$ and $g_{2}$.

Since $0 \notin I$, the cases for $w$ that ended or started with 1 are one of the cases in the table. Therefore, the table completes the proof.

Since $\Sigma(I)$ does not have any isolated point, then Theorems 1 and 2 prove that $(\Sigma(I), \sigma)$ is Devaney chaotic.

The increasing gap shift is strongly blending as stated by the following theorem.

\section{Theorem 3. $(\Sigma(I), \sigma)$ is strongly blending.}

Proof. Let $C_{w}$ and $C_{v}$ be two basic open subsets of $\Sigma(I)$ for two allowable blocks $w=w_{0} w_{1} \ldots w_{l-1}$ and $v=v_{0} v_{1} \ldots v_{k-1}$ with lengths $l$ and $k$, respectively. We consider all possibilities of $w$ and $v$ by looking at its possibilities of 1 to be suffix of the blocks.

The first case is whenever $w 1$ and $v 1$ are both allowed. Let $n \in \mathbb{N}$ such that $n>l, n>k$ and $n=l+r_{1}, n=k+r_{2}$ for some $r_{1}, r_{2} \in \mathbb{N}$. Let us choose $u$ to be an allowed block in $\Sigma(I)$ such that $1 u$ is also allowed and let $\mathbf{z} \in C_{u}$. Then, take $\mathbf{x}=w \underbrace{11 \ldots 11}_{r_{1}} \mathbf{z} \in C_{w}$ and $\mathbf{y}=\underbrace{11 \ldots 11}_{r_{2}} \mathbf{z} \in C_{v}$. Then, $\sigma^{l+r_{1}}(\mathbf{x})=\sigma^{n}(\mathbf{x})=\mathbf{z}$ and $\sigma^{k+r_{2}}(\mathbf{y})=\sigma^{n}(\boldsymbol{y})=\mathbf{z}$. Thus, $\mathbf{z} \in$ $\sigma^{n}\left(C_{w}\right) \cap \sigma^{n}\left(C_{v}\right)$. Therefore, $C_{u} \subseteq \sigma^{n}\left(C_{w}\right) \cap \sigma^{n}\left(C_{v}\right)$.

The second case is whenever $w 1$ is allowed but not $v 1$. Without loss of generality, we assume that $v=$ $v_{0} v_{1} \ldots v_{k-c_{2}-2} 1 \underbrace{100 \ldots 00}_{c_{2}}$, where $c_{2} \notin I$ and $c_{2}+g_{2} \in I$ for some integer $g_{2}$. Let $v^{\prime}=\underbrace{00 \ldots 00}_{g_{2}}$. Now, $w 1$ and $v^{\prime} 1$ are allowed. By the first case, there exists an integer $n>0$ such that $\sigma^{n}\left(C_{w}\right) \cap \sigma^{n}\left(C_{v^{\prime}}\right)$ contains a nonempty open subset. Then, $\sigma^{n}\left(C_{w}\right) \cap \sigma^{n}\left(C_{v}\right)$ must also contain the nonempty open subset.

The last case is neither $w 1$ and $v 1$ are allowed. Without loss of generality, let $w=w_{0} w_{1} \ldots w_{l-c_{1}-2} 1 \underbrace{00 \ldots 00}_{c_{1}}$ and $v=$ $v_{0} v_{1} \ldots v_{k-c_{2}-2} 1 \underbrace{00 \ldots 00}_{c_{2}}$, where $c_{1}, c_{2} \notin I$ and $c_{1}+g_{1} \in I$, $c_{2}+g_{2} \in I$ for some integer $g_{1}$ and $g_{2}$. Let $w^{\prime}=\underbrace{w 00 \ldots 00}_{g_{1}}$ and $v^{\prime}=\underbrace{00 \ldots 00}_{g_{2}}$ such that $w^{\prime} 1$ and $v^{\prime} 1$ are both allowed. By the first case, there exists an integer $n>0$ such that $\sigma^{n}\left(C_{w^{\prime}}\right) \cap \sigma^{n}\left(C_{v^{\prime}}\right)$ contains a nonempty open subset. Then, $\sigma^{n}\left(C_{w}\right) \cap \sigma^{n}\left(C_{v}\right)$ must also contain the nonempty open subset.

Therefore, $(\Sigma(I), \sigma)$ is strongly blending.

However, $\Sigma(I)$ is not l.e.o. as given in the following.

Theorem 4. $(\Sigma(I), \sigma)$ is not locally everywhere onto.

Proof. On the contrary, suppose that $(\Sigma(I), \sigma)$ is l.e.o. Then, for every basic open subset $C_{u} \subset \Sigma(I)$, there exists an integer $k \in \mathbb{N}$ such that $\sigma^{k}\left(C_{u}\right)=\Sigma(I)$. Let $C_{u}=C_{1}$ and $\sigma^{n}\left(C_{1}\right)=$ $\Sigma(I)$ for some $n \in \mathbb{N}$. Let $m>n+1$. Since $a_{m+1}=a_{m}+m+1$, then, from the way we define $I, m+1$ is the smallest integer $r$ such that $a_{m}+r \in I$. Now let $\mathbf{s} \in \Sigma(I)$ such that $\mathbf{s}=$ $\underbrace{00 \ldots 001}_{c} s_{c+1} s_{c+2} s_{c+3} \ldots$ where $c=a_{m}+1$. Since $\sigma^{n}\left(C_{1}\right)=$ $\Sigma(I)$, then there exists $\mathbf{t} \in C_{1}$ such that $\sigma^{n}(\mathbf{t})=\mathbf{s}$. Then, $\mathbf{t}=1 t_{1} t_{2} \ldots t_{n-1} \underbrace{00 \ldots 00}_{c} 1 s_{c+1} s_{c+2} s_{c+3} \ldots=1 t_{1} t_{2} \ldots t_{n-1} \mathbf{s}$. Since $\mathbf{t} \in \Sigma(I)$, then there exists an integer $l \leq n-1$ such that $\mathbf{t}=1 t_{1} t_{2} \cdots \underbrace{100 \ldots 0000 \ldots 00}_{l} 1 s_{c+1} s_{c+2} s_{c+3} \ldots$ and $l+c \in I$. So $l+c=a_{j}$ for some $j \in \mathbb{N}_{0}$. So $l+a_{m}+1=a_{j}$. Since $m+1$ is the smallest integer $r$ such that $a_{m}+1+r \in I$, then $l \geq m$. But $m>n+1$ which implies $l>n+1$, and therefore it contradicts to $l \leq n-1$. Therefore, $(\Sigma(I), \sigma)$ is not l.e.o. 
TABLE 2: Cases for block $w$.

\begin{tabular}{|c|c|}
\hline Block $\boldsymbol{w}$ of length $\boldsymbol{l}$ & Periodic point $\mathbf{x} \in C_{w}$ \\
\hline$w=\underbrace{00 \cdots 001}_{p_{1}} w_{p_{1}+1} \cdots w_{l-p_{2}-2} 1 \underbrace{00 \cdots 00}_{p_{2}}$ & $\mathbf{x}=\overline{w \underbrace{11 \cdots 11}_{n}}$ \\
\hline$w=\underbrace{00 \cdots 001}_{p_{1}} w_{p_{1}+1} \cdots w_{l-c_{2}-2} 1 \underbrace{00 \cdots 00}_{c_{2}}$ & $\mathbf{x}=\overline{\overline{w \underbrace{00 \cdots 0011 \cdots 11}_{g_{2}}}}$ \\
\hline$w=\underbrace{00 \cdots 001}_{c_{1}} w_{c_{1}+1} \cdots w_{l-p_{2}-2} \underbrace{100 \cdots 00}_{p_{2}}$ & $\mathbf{x}=\overline{\overline{w 11 \cdots 1100 \cdots 00}}$ \\
\hline$w=\underbrace{00 \cdots 001}_{c_{1}} w_{c_{1}+1} \cdots w_{l-c_{2}-2} 1 \underbrace{100 \cdots 00}_{c_{2}}$ & $\mathbf{x}=\overline{\overline{w \underbrace{00 \cdots 0011 \cdots 11}_{g_{2}} \underbrace{100 \cdots 00}_{n}}}$ \\
\hline $\boldsymbol{w}=\underbrace{00 \ldots 00}_{l}$, where $l \in I$ & $\mathbf{x}=\overline{w \underbrace{11 \cdots 11}_{n}}$ \\
\hline $\boldsymbol{w}=\underbrace{\mathbf{0 0 \cdots 0 0}}_{l}$, where $\boldsymbol{l} \notin I$ & $\begin{array}{c}\mathbf{x}=\overline{w \underbrace{00 \cdots 0011 \cdots 11}_{g}}, \text { where } \\
g \in \mathbb{N} \text { such that } l+g \in I\end{array}$ \\
\hline
\end{tabular}

\section{Conclusion}

Topologically transitive is one of the two components of Devaney chaos. In this paper we look at three chaos notions which are stronger than transitivity: totally transitive, topologically mixing, and l.e.o. It is generally known that totally transitive, topologically mixing, and locally everywhere onto are equivalent on shift of finite type. It has been discussed earlier that the sigma star is a shift of infinite type which satisfies totally transitive but not topologically mixing. We have presented another example of shift of infinite type, the increasing gap shift which has topologically mixing property but not locally everywhere onto. Therefore, these two counterexamples show that these three chaos notions are not equivalence on shift of infinite type. In addition, we have also shown that the increasing gap shift is strongly blending. The shift of finite type with forbidden blocks 00 and 10 is also strongly blending but not l.e.o. [17]. Therefore, strongly blending and l.e.o. are not equivalence on all shift spaces.

Specification property is another chaos notion which is equivalent to l.e.o., mixing, and totally transitive on shift of finite type. However, as far as we are concerned this relation on shift infinite type is still unknown.

\section{Data Availability}

There is no data used in this article.

\section{Conflicts of Interest}

The authors of this manuscript declare that there are no conflicts of interest regarding the publication of this paper.

\section{Acknowledgments}

The authors would like to thank Universiti Kebangsaan Malaysia and Center for Research and Instrumentation (CRIM) for the financial funding through FRGS/1/2017/ STG06/UKM/02/2.

\section{References}

[1] R. L. Devaney, An Introduction to Chaotic Dynamical Systems, Allan M. Wylde, 1989.
[2] J. Banks, J. Brooks, G. Cairns, G. Davis, and P. Stacey, "On Devaney's definition of chaos," The American Mathematical Monthly, vol. 99, no. 4, pp. 332-334, 1992.

[3] J. L. G. Guirao, D. Kwietniak, M. Lampart, P. Oprocha, and A. Peris, "Chaos on hyperspaces," Nonlinear Analysis: Theory, Methods \& Applications, vol. 71, no. 1-2, pp. 1-8, 2009.

[4] C. Thomson, A hierarchy of chaotic topological dynamics, [MSc thesis], Davidson College, 2013.

[5] J. Li and X. D. Ye, "Recent development of chaos theory in topological dynamics," Acta Mathematica Sinica, vol. 32, no. 1, pp. 83-114, 2016.

[6] A. Fan, L. Liao, and J. Peyriére, "Generic points in systems of specification and Banach valued Birkhoff ergodic average," Discrete and Continuous Dynamical Systems - Series A, vol. 21, no. 4, pp. 1103-1128, 2008.

[7] A. Crannell, "A chaotic, non-mixing subshift," Discrete and Continuous Dynamical Systems - Series A, vol. 43, no. 1, pp. 195202, 1998.

[8] L. Alseda, M. Delrio, and J. Rodriguez, "Transitivity and dense periodicity for graph maps," Journal of Difference Equations and Applications, vol. 9, no. 6, pp. 577-598, 2003.

[9] M. Denker, C. Grillenberger, and K. Sigmund, Ergodic Theory on Compact Spaces, Springer-Verlag, New York, NY, USA, 1976.

[10] C. D. Syahida and C. Good, "On devaney chaos and dense periodic points: period 3 and higher implies chaos," The American Mathematical Monthly, vol. 122, no. 8, pp. 773-780, 2015.

[11] B. Malouh and C. D. Syahida, "Implication diagram of five chaos characterizations: a survey on compact metric space and shift of finite type," Journal of Quality Measurement and Analysis, vol. 13, no. 1, pp. 15-23, 2017.

[12] M. Vellekoop and R. Berglund, "On interval, transitivity = chaos," The American Mathematical Monthly, vol. 101, no. 4, pp. 353-355, 1994.

[13] B. Malouh, C. D. Syahida, and C. Good, "Dense periodicity property and devaney chaos on shift spaces," International Journal of Mathematical Analysis, vol. 10, pp. 1019-1029, 2016.

[14] S. Ruette, Chaos on the Interval, vol. 67 of University Lecture Series, American Mathematical Society, Providence, RI, USA, 2017.

[15] S. Baker and A. E. Ghenciu, "Dynamical properties of S-gap shifts and other shift spaces," Journal of Mathematical Analysis and Applications, vol. 430, no. 2, pp. 633-647, 2015. 
[16] A. D. Dawoud and J. Somaye, "Dynamics and topology of Sgap shifts," Topology and its Applications, vol. 159, no. 10-11, pp. 2654-2661, 2012.

[17] B. Malouh and C. D. Syahida, "The dynamics of 1-step shifts of finite type over two symbols," Indian Journal of Science and Technology, vol. 9, no. 46, pp. 1-5, 2016. 


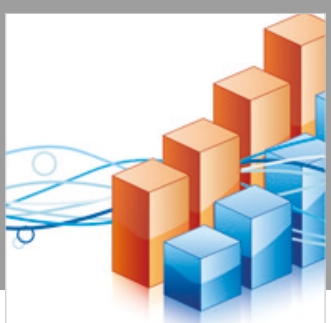

Advances in

Operations Research

\section{-n-m}
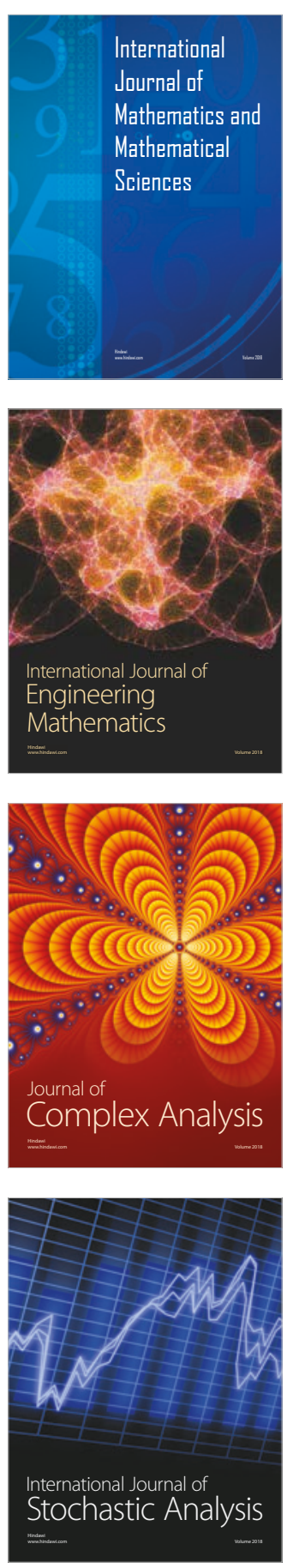
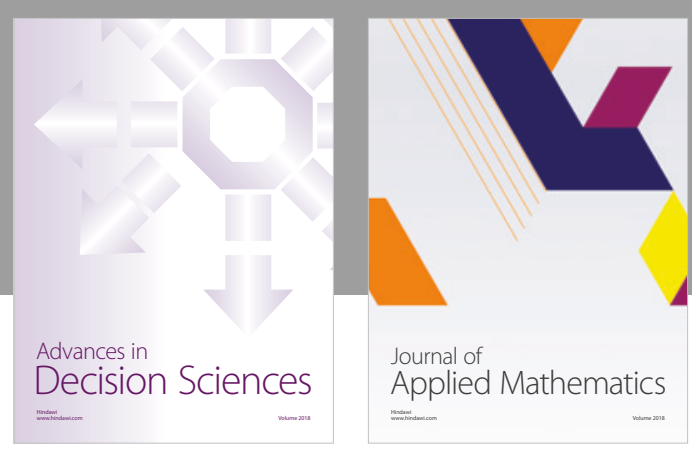

Journal of

Applied Mathematics
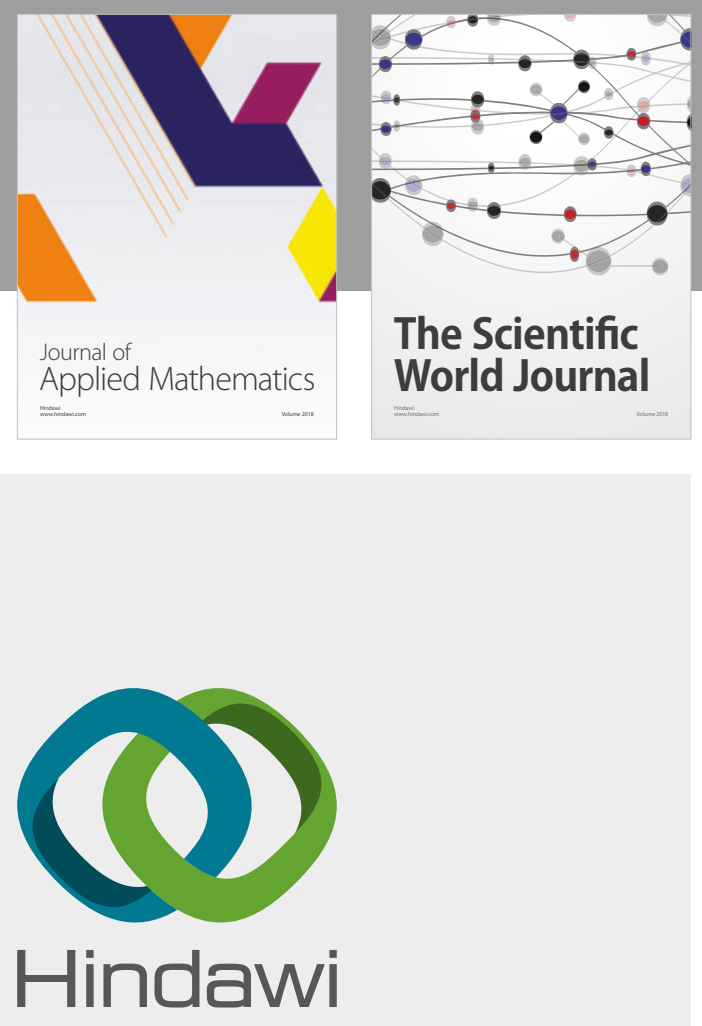

Submit your manuscripts at

www.hindawi.com

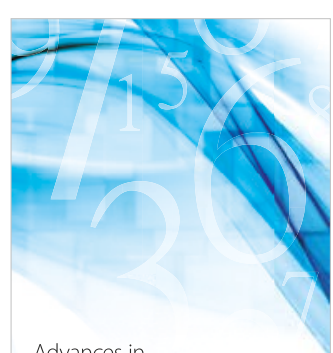

Advances in
Numerical Analysis
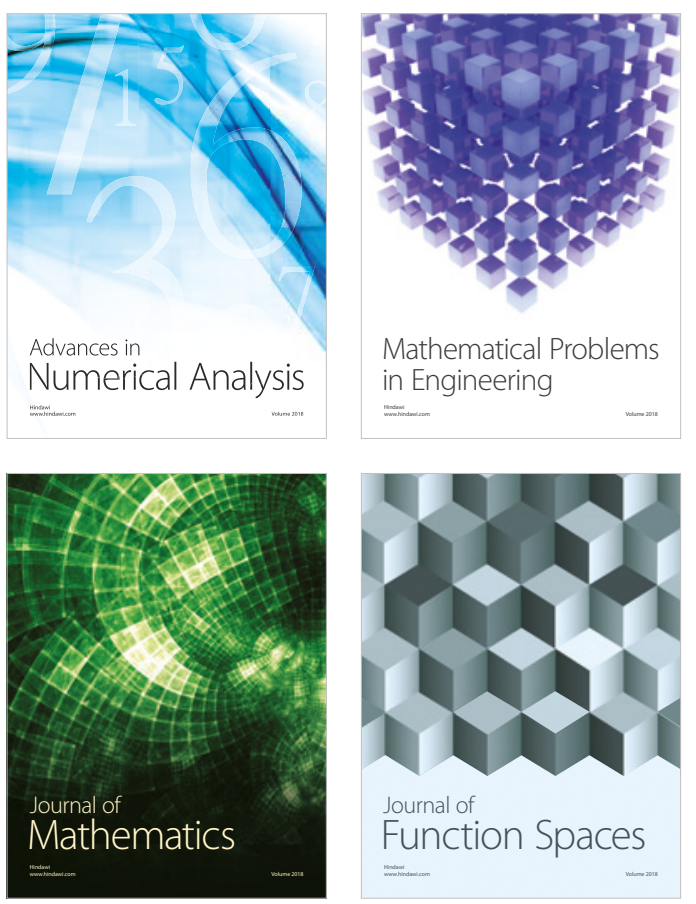

Mathematical Problems in Engineering

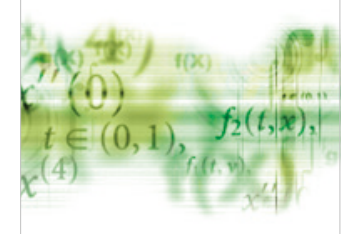

International Journal of

Differential Equations

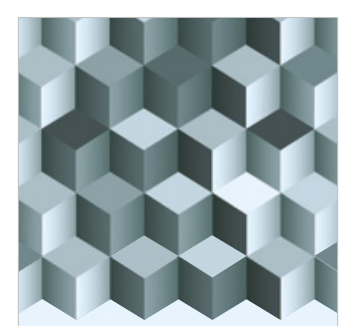

Journal of

Function Spaces

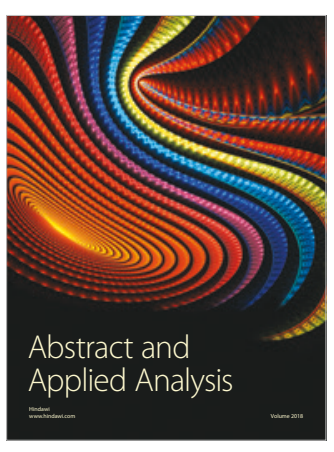

The Scientific

World Journal

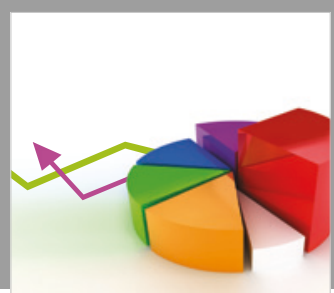

Journal of

Probability and Statistics
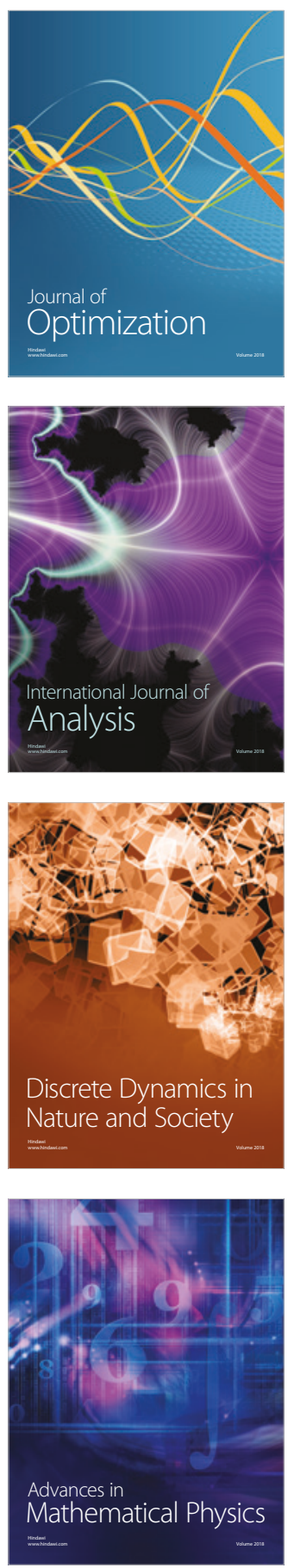